\title{
Fehlendes Fachwissen an entscheidenden Stellen
}

\author{
Das Interview führte: Matthias Scholer \\ Chefredaktor SÄZ
}

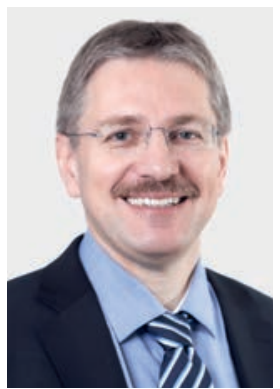

Carlos Quinto,

Departementsverantwortlicher «Public Health, Gesundheitsberufe und Heilmittel» bei der FMH

Die Schweiz bereitet sich seit 1995 auf eine Influenza-Pandemie vor. Dazu gehört auch die Publikation «Influenza-Pandemieplan Schweiz», welche vor zwei Jahren überarbeitet wurde. Viele Aspekte in diesem Planungsinstrument fanden auch bei der derzeitigen SARS-CoV-2-Pandemie Anwendung. Doch bei einigen Playern im Gesundheitswesen erfuhr der Pandemieplan geringe Aufmerksamkeit und wurde schubladisiert.

\section{Wie haben Sie die Wochen seit der Ausrufung der «ausserordentlichen Lage» in ihrer Tätigkeit als Hausarzt erlebt?}

In einer ersten Phase spürten wir eine grosse Verunsicherung. Dies zeigte sich in vermehrten Fragen gegen Ende der Konsultation oder am Telefon. Nach dem Lockdown folgte die Beschränkung auf das Nötigste. Je länger diese Phase andauerte, umso mehr meldeten sich Seniorinnen und Senioren telefonisch, die sehr unter der Isolation litten. Seit der Lockerung der Massnahmen steigt die Anzahl Sprechstundentermine kontinuierlich an. Dabei merken wir, dass sich gewisse medizinische Probleme in Folge der eingeschränkten Bewegungsfreiheit zugespitzt haben. So stellten wir beispielsweise fest, dass sich die Blutzuckerwerte von Diabetikern aufgrund des Bewegungsmangels verschlechterten.

In der Publikation "Influenza-Pandemieplan Schweiz 2018» des Bundesamtes für Gesundheit wird den Hausarztpraxen empfohlen, welches Schutzmaterial sie auf Lager haben sollten. Hatten Sie diese Empfehlung in Ihrer Praxis vor der Pandemie umgesetzt? Ja, aber ich muss dazu sagen, dass ich vor drei Jahren das Glück hatte, an einer Sitzung im Generalsekretariat der FMH mit Vertretern des Bundesamts für wirtschaftliche Landesversorgung BWL teilgenommen zu haben. Dabei wurde der Pandemieplan im Detail diskutiert. Im Nachgang setzten wir in unserer Praxis die Empfehlungen bezüglich Schutzmaterial um. Ich weiss nicht, wie viele Kolleginnen und Kollegen einen solchen Vorrat angelegt haben, aber meines Erachtens bestand das grösste Defizit bei Kantonen, Spitälern sowie Alters- und Pflegeheimen, die, wie mir eine Kantonsvertreterin schilderte, den Pandemieplan häufig ohne Umsetzung schubladisierten. Der Grund war wohl, dass es sich dabei um Vorhalteleistungen handle, die Kosten verursachten.

Der Bundesrat wird seit März von einer «science task force" unterstützt. Ist dies auch als Zeichen zu werten, dass das Wissen rund um Public Health und Medizin auf Stufe der Entscheidungsträger untervertreten ist? Tatsächlich sind die Leitungsgremien von Behörden auf kantonaler und nationaler Ebene «medizinfrei». In den entscheidenden Positionen sind keine Ärzte, Veterinäre, Pharmazeuten oder weitere ähnliche Berufsgattungen vertreten, die in Pandemiesituationen ihr Fachwissen einbringen können. Viele Stellen im Gesundheitsbereich wurden in letzter Zeit zunehmend nach politischen und nicht nach fachlichen Kriterien besetzt. Im Bereich Public Health stufte man die kantonsärztlichen Dienste herab. Sie haben nun mehr übergeordnete Administrationsstufen zu bedienen. Zudem wurden ihre personellen Ressourcen gekürzt. In Folge konnten die Dienste zu Beginn der Pandemie ihre Aufgaben nicht optimal ausführen. Das Gleiche gilt auf Bundesebene für das Bundesamt für Gesundheit. Dort gibt es ausgezeichnete Public-Health-Expertinnen und -Experten. Sie sind aber alle hierarchisch tief angesiedelt. Bis das Fachwissen zu den Entscheidungsträgern vordringt, vergeht wertvolle Zeit. Das ist in Pandemiesituationen deletär.

Ist dieses fehlende Wissen in den Entscheidungsgremien auch der Grund, weshalb der Pandemieplan in vielen Schubladen verschwand?

Der Pandemieplan erfuhr bei einigen Gremien der Bundesverwaltungen keine oder nur geringe Wert- 
schätzung. Wenn ich Ökonom oder Jurist wäre, würde ich einen Pandemieplan auch schubladisieren, weil ich eine zu grosse Distanz zur Materie hätte. Wenn man in einem Gesundheitswesen den Patienten ins Zentrum stellen möchte, müssen auch die Professionen, bei denen der Patient im Zentrum steht, auf allen Hierarchiestufen genügend vertreten sein. Juristen, Ökonomen und weitere nicht medizinische Berufe können helfen, die Rahmenbedingungen mitzudefinieren, aber nicht die Medizin selbst. Im Public-Health-Bereich hingegen ist interdisziplinär zu arbeiten, wobei «Health» eine zentrale Rolle einnimmt.

Viele kritisieren die Massnahmen retrospektiv als zu strikt, schliesslich sei die Mortalität bei Covid-19 nicht höher als bei einer schweren saisonalen Grippe. Wie schätzen Sie solche Vergleiche ein?

Die Mortalität ist ein eindeutiger Messwert bei einer Pandemie. Aber die Mortalität kann erst nach einer Pandemie genau bestimmt werden. Die Mortalität alleine ist der falsche Indikator, um zu beurteilen, ob der Lockdown gerechtfertigt war. Dazu muss man den disease burden als Basis nehmen. Die Krankheitslast hängt wiederum stark von der Prävalenz ab. Wie lange sind die Patienten krank? Wie lange benötigen sie Spitalpflege? Ist eine Rehabilitation nötig? Gibt es bleibende Schäden? Dies sind die Faktoren, welche die Ressourcen eines Gesundheitswesens beanspruchen.

Bei der Spanischen Grippe betrug die Todesrate der zweiten Welle phasenweise ein Vielfaches der Mortalität während der ersten Welle. Droht uns ein solches Szenarium auch bei Covid-19?

Der Schweregrad der nachfolgenden Wellen wird massgeblich davon abhängen, ob es beim Virus zu Mutationen kommt und wie die Resilienz der Bevölkerung ist. Gut möglich, dass sich infolge beispielsweise mangelnder Bewegung, schlechterer Ernährung, psychischer Belastungen oder ökonomischer Faktoren der Gesundheitszustand der Bevölkerung generell verschlechtert hat. Eines ist sicher: Wir werden die Situation erst unter Kontrolle haben, wenn neben Medikamenten auch ein Impfstoff zur Verfügung steht.

Arzneimittel und Impfstoffe werden kaum mehr in der Schweiz produziert. Denken Sie, dass die Corona-Pandemie in diesem Bereich zu einem Umdenken führen wird? Wenn wir die Abhängigkeit von aussereuropäischen Ländern reduzieren wollen, gibt es nur zwei Möglichkeiten. Entweder wird wieder vermehrt in Europa pro- duziert. Dann ist unsere Aussenpolitik gefordert, damit wir einen niederschwelligen Zugang $\mathrm{zu}$ den Produkten und die Möglichkeit von Kooperationen erhalten. Oder wir stellen Arzneimittel und Impfstoffe im grossen Stil in der Schweiz her. Aus ökonomischen Gründen müssen wir dann jedoch zumindest Europa mit diesen Produkten beliefern können. Für eine gute Lösung braucht es Gespräche, an denen die Ärzteschaft, die Apothekerschaft, das Bundesamt für Gesundheit, swissmedic und weitere involvierte Player konstruktiv nach einer Lösung suchen.

\section{Bekommen wir Prämienzahler die Folgen der} Betriebsausfälle von Spitälern und Praxen zu spüren? Die Betriebsausfälle und Mehrkosten bei den Spitälern sind primär ein durch den Bund, aus nachvollziehbaren Gründen, ausgelöstes Problem der Kantone. Deshalb erwarte ich den grössten Kostenschub bei den Steuern und nicht bei den Krankenkassenprämien, ausser es wird querfinanziert. Wenn es zu massiven Mehrkosten bei den Krankenversicherungen käme, wäre das schwer erklärbar oder politisch gewollt. Aufseiten der privaten Praxen können die behördlich verordneten Verluste vielleicht durch den Nachholbedarf infolge aufgestauter Abklärungen, Beratungen und Behandlungen kompensiert werden.

Ich erwarte den grössten Kostenschub bei den Steuern und nicht bei den Krankenkassenprämien.

\section{Sie engagieren sich neben Ihrer Arbeit als Hausarzt standespolitisch und sind Departementsverantwortli- cher «Public Health, Gesundheitsberufe und Heilmittel» bei der FMH. Woher kommt dieses breite Engagement?} Der Schlüsselmoment war ein Auftritt des Kantonsarztes ausser Plan, in einer Vorlesung. Es ging um Aids, an dem gerade in der Schweiz die Erkrankungsfälle zunahmen. Über das HI-Virus und die durch es ausgelöste Epidemie war zu Beginn meines Medizinstudiums in den 1980er Jahren noch nicht viel bekannt. Dazumal herrschte eine ähnliche Stimmung wie heute. Nach der Vorlesung wurde mir klar, dass der Beruf des Arztes immer auch eine soziale und politische Dimension beinhaltet. Mein Interesse für diese Dimensionen war geweckt. Spätere berufliche Begegnungen sowie Erfahrungen beeinflussten mich in der Wahl meiner Berufsfelder Hausarztmedizin und Public Health. Beide sind bis heute die Quelle meines Engagements. 